\title{
FASTEST CURVES AND TOROIDAL BLACK HOLES
}

\author{
E. WOOLGAR \\ Department of Mathematics, University of Saskatchewan \\ 106 Wiggins Ave., Saskatoon, SK, Canada S7N 5E6 \\ E-mail:woolgar@math.usask.ca
}

\begin{abstract}
We discuss an apparent paradox (and conjectured resolution) of Jacobson and Venkataramani concerning "temporarily toroidal" black hole horizons, in light of a recent connectivity theorem for spaces of complete causal curves. We do this in a self-contained manner by first reviewing the "fastest curve argument" which proves this connectivity theorem, and we note that active topological censorship can be derived as a corollary of this argument. We argue that the apparent paradox arises only when one dispenses with the invariant viewpoint provided by the connectivity theorem in favour of an observer-dependent description. Finally, we discuss an alternative to fastest curve arguments, which can be used to construct a self-contradictory null line in certain spacetimes violating topological censorship. These arguments may shed light on the relationship between topological and cosmic censorship.
\end{abstract}

1. Introduction. The study of Lorentzian geometry has progressed, at least in part, by adapting tools found to be effacacious within Riemannian geometry, though one must keep in mind that such adaptation is not always useful nor even always possible. Consider the notable example of the Hopf-Rinow theorem. In part, this theorem states that geodesic completeness, an important instrument of global geometrical analysis, is equivalent to Cauchy completeness and to finite compactness, so it is not surprising that these last two properties provide useful tools in Riemannian geometry. No analogue of this aspect of Hopf-Rinow is available in Lorentzian geometry. This does not mean that Lorentzian geometry is less rich, for one has new tools, which make use of causal relations. The role of Riemannian concepts like Cauchy completeness or finite compactness is assumed by "causal regularity conditions" like global hyperbolicity or causal continuity, while geodesic completeness is replaced by the more specialized notions of timelike and null geodesic completeness.

The Hopf-Rinow theorem also states that a complete Riemannian metric implies a minimal curve between any two points. There are Lorentzian analogues of this part of

1991 Mathematics Subject Classification: Primary 53C50; Secondary 83C75.

The paper is in final form and no version of it will be published elsewhere. 
the theorem. An oft-cited one is the existence of maximal timelike curves joining any pair of timelike-related points in a globally hyperbolic spacetime. It is less often noted that there is also an analogous result for null curves, which one may express in the following way. Let there exist causal curves from both $x$ and $y$ to $z$, but not from $x$ to $y$, and let $\zeta$ be a causal curve from $y$ to $z$. Assuming global hyperbolicity, then there will exist a fastest causal curve from $x$ to $\zeta$. A curve $\gamma$ from $x$ to $\zeta$ is fastest if there is a $w \in \gamma \cap \zeta$ such that $s \in J^{+}(x) \cap \zeta$ implies $s \geq w$; in other words, $w$ lies on a causal curve from $x$ and is the infimum, in the induced causal order on $\zeta$, of those points that can be so reached from $x$. Then $\gamma$ lies on $\partial I^{+}(x)$ from $x$ to $w$; in consequence, it is a null geodesic and has no points conjugate to $x$ along it (before $w$; we will not insist that fastest curves end at $w$; then the definition then places no conditions on any segment of $\gamma$ lying beyond $w$ ).

But consider a spacetime that is asymptotically flat, so that it admits a boundaryat-infinity $\mathcal{I}$. As is standard, we assume that $\mathcal{I}$ is the null cone of a point $i^{0}$ representing spatial infinity, or perhaps it is a disjoint union of many such cones. If $x \in \mathcal{I}^{-}$and $\zeta$ lies on $\mathcal{I}^{+}$, then a fastest curve $\gamma$ from $x$ to $\zeta$ would have to be a conjugate-point-free null geodesic which leaves $\mathcal{I}^{-}$, travels through spacetime, and returns to $\mathcal{I}^{+}$. The segment of $\gamma$ that lies within spacetime would be infinite, as measured by affine parameters defined by the spacetime metric; it's a complete null line. ${ }^{1}$ But if one assumes an energy condition, even a very mild "integrated" energy condition, and the generic condition, then every null geodesic must have a pair of conjugate points lying within finite affine distance from each other. As a consequence, null lines are forbidden, and there is no fastest curve from $x$ to $\zeta$.

Recall now another result from Riemannian geometry, that if a complete Riemannian manifold has more than one asymptotic end it admits a complete line (in this case, a complete geodesic realizing the Riemannian distance between any pair of its points). By way of analogy, one might therefore speculate that globally hyperbolic Lorentzian manifolds with more than one component of $\mathcal{I}$ would admit complete null lines joining these components. But when the physically reasonable assumptions used above hold, we have said complete null lines are forbidden; these postulated null lines cannot actually occur.

In the Riemannian case, one can place a condition on the sign of curvature components which will forbid complete lines, implying in turn that such manifolds have only one end. However, in the Lorentzian case reasonable energy conditions are not strong enough to allow for an argument to rule out complete spacelike lines, and in consequence multiple ends are not forbidden. Then what mechanism acts to prevent the completion of the null lines? A theorem of Gannon states that if a globally hyperbolic, asymptotically flat spacetime obeys a suitable energy condition and admits a Cauchy surface with more than one asymptotic end (whence there are multiple components of $\mathcal{I}$ ), or if the spacetime has a covering spacetime with these properties, then it is null geodesically incomplete [5]. Here

\footnotetext{
${ }^{1} \mathrm{~A}$ line in a Lorentzian manifold is an inextendible geodesic that realizes the Lorentzian distance between any pair of its points. In the case of a null line, this distance is always zero. A line cannot have conjugate points, since a geodesic with conjugate points does not realize the Lorentzian distance between points that lie beyond a conjugate pair.
} 
the global hyperbolicity assumption may well be tantamount to a censorship condition suggesting that, if the manifold is inextendible, there would be horizons which cloak the incomplete geodesics. In this case at least, one can postulate that these horizons separate the components of $\mathcal{I}$, preventing null lines, or any causal curves, from joining different components, in accord with the arguments concerning $\zeta$ and $x$ above. This scenario is known to be correct, in consequence of the active topological censorship theorem, first proved by Friedman, Schleich, and Witt [3]. They did not employ fastest curves to prove this theorem, although they were aware that a proof along these lines could be given, and they sketched an argument.

Indeed, in the Lorentzian case, direct application of these "fastest curve" arguments produces a result that is actually more powerful than a topological censorship theorem, and concerns not spacetime topology but the topology of the space $C(p, q)$ of causal curves that join $p \in \mathcal{I}^{-}$to $q \in \mathcal{I}^{+}$. This result, that the space $C(p, q)$ is connected, was obtained by Penrose, Sorkin, and Woolgar, and will receive a full discussion in a forthcoming paper [9], wherein it will be given an interpretation as a positive energy theorem (cf. [8]).

In this paper, the theorem is instead applied to the question of topological censorship and a related issue of horizon topology. In particular, we treat certain questions raised by Jacobson and Venkataramani [7]. They speculated that such a theorem - more precisely, a very similar theorem - should exist and would have an interesting application to the case of a "temporarily toroidal" black hole horizon. They remarked that causal curves could appear to thread this torus and would therefore appear to violate the theorem. They further suggested that this apparent paradox could be resolved if these curves also appeared to be "time-delayed."

Herein we show that the connectivity theorem, although perhaps subtlely weaker than the theorem that the authors of [7] had in mind, may be applied in essentially the same manner as they discussed, thereby producing the same apparent paradox. The theorem is compelling evidence that no causal curve actually threads the torus formed by the horizon; then the appearance of such threading, and concomitant time-delay effect, is the result of a non-invariant description based in a choice of slicing for spacetime.

In Section 2, we borrow heavily from [9] to present the proof of connectedness of $C(p, q)$, which we will need for later reference. Peripherally, as part of the proof, we show that the active topological censorship theorem of Friedman, Schleich, and Witt [3] can be thought of in essence as a corollary of the connectivity theorem. ${ }^{2}$ The contents of this section are not new, and are provided in order to inform the subsequent discussion in Section 3. The demonstration of the censorship theorem largely parallels an argument outlined in [3], wherein it was suggested as an alternative to the main demonstration of the censorship theorem given there (which did not use "fastest curves"). In Section 3 , the connectivity theorem is applied to the question of temporarily toroidal horizons, in order to clarify the aforementioned apparent paradox. In the final section, we outline an argument which is in many ways analogous to the fastest curve argument, but uses

\footnotetext{
${ }^{2}$ It is not precisely a corollary, since the [3] result relies on a different focusing condition than the one in use here.
} 
null geodesic completeness instead of global hyperbolicity, and which has potential for clarifying the underlying structure responsible for enforcing topological censorship.

2. The connectivity theorem and its censorship corollary. To give meaning to an assertion concerning the connectedness of a space of curves, we must first say what the topology is. Let $x, y$ be two points in a spacetime $\left(\mathcal{M}, g_{a b}\right)$ and let $C(x, y)$ be the space whose elements are the causal curves beginning at $x$ and ending at $y$. Let $\mathcal{O}$ be any $\mathcal{M}$-open set. Then we define the set $\mathcal{B}(\mathcal{O}) \subseteq C(x, y)$ by

$$
\mathcal{B}(\mathcal{O})=\{\gamma \mid \gamma \in C(x, y), \gamma \subseteq \mathcal{O}\}
$$

Thus, the causal curve $\gamma$ from $x$ to $y$ belongs to the subset $\mathcal{B}(\mathcal{O})$ of $C(x, y)$ iff $\gamma$ is contained within the open set $\mathcal{O}$ in $\mathcal{M}$. We define the topology on $C(x, y)$ to be that topology whose open sets are arbitrary unions of all such basis sets $\mathcal{B}(\mathcal{O})$, where $\mathcal{O}$ is open in $\mathcal{M}$. This is a standard choice, at least when $\mathcal{M}$ is strongly causal (we will in fact use this topology below only when $\mathcal{M}$ is globally hyperbolic, and thus strongly causal). We now state the theorem and then comment on the assumptions.

THEOREM 2.1. Let a spacetime $\left(M, g_{a b}\right)$ be asymptotically flat with boundary-at-infinity $\widehat{\mathcal{I}}$ comprised of a disjoint union of one or more components, and let $\mathcal{I}$ be one such component. Let $D=I^{+}\left(\mathcal{I}^{-}\right) \cap I^{-}\left(\mathcal{I}^{+}\right)$be the domain of outer communications belonging to the component $\mathcal{I}$, and assume that $D \cup \mathcal{I}$ can be embedded in an open globally hyperbolic "extended spacetime" $\left(\widetilde{D}, \widetilde{g}_{a b}\right)$ (the embedding is a conformeomorphism on the image of $\left.\left(M, g_{a b}\right)\right)$. Let $D$ be null focusing.

(i) Then the space $C(p, q)$ of causal curves from $p \in \mathcal{I}^{-}$to $q \in \mathcal{I}^{+}$is connected, and

(ii) active topological censorship holds for $D$.

Here active topological censorship will be taken to mean that every causal curve from $p \in \mathcal{I}^{-}$to $q \in \mathcal{I}^{+}$can be deformed to a null generator of $\mathcal{I}$. The deformation proceeds through a homotopy on the space of continuous curves, but not necessarily on the space $C(p, q)$ of causal curves. This space refers to the causal curves in the extended spacetime, and so includes the null generator of $\mathcal{I}$ from $p$, through $i^{0}$ (where it need not be smooth), to $q .{ }^{3}$

The global hyperbolicity of $\left(\widetilde{D}, \widetilde{g}_{a b}\right)$ implies global hyperbolicity of $\left(D, g_{a b}\right)$. Technical issues concerning the presence in the larger globally hyperbolic spacetime of nondifferentiable points, such as is the case with $i^{0}$ when the mass is non-zero, are handled in $[11]$.

\footnotetext{
${ }^{3}$ The theorem will apply even for asymptotically flat $(2+1)$-dimensional spacetimes, but in this case Cauchy surfaces do not have so-called simply connected neighbourhoods of infinity. As a consequence, $C(p, q)$ would not be connected if the null generator of $\mathcal{I}$ were not included in it, and the homotopy referred to by the censorship result might necessarily pass through curves that meet $\mathcal{I}$. In the $(3+1)$-dimensional asymptotically flat case, simply connected neighbourhoods of infinity are assumed, and our statement that a curve should be homotopic to a generator of $\mathcal{I}$ becomes equivalent to the usual condition that a curve is homotopic within spacetime to a curve within such a simply connected neighbourhood of $\mathcal{I}$.
} 
A domain of spacetime is null focusing if every complete null geodesic in it possesses a pair of conjugate points. Focusing lemmata guarantee that this is always the case whenever a suitable energy condition and the null generic condition hold. ${ }^{4}$ Examples of suitable conditions are the Average Null Energy Condition (which requires non-negativity of an integral of a Ricci curvature component along each null geodesic) [12] and the Borde Energy Condition (which is a requirement on integrals of Ricci components along finite portions of each null geodesic) [1].

Now we are ready to give the proof. The proof of part $(i)$ is abstracted from [9], wherein it is given a different interpretation. The idea to interpret it as below grew out of a suggestion that appears in [7]. The proof of part (ii) closely corresponds to an argument first outlined in [3].

Proof. Let $\Lambda^{+}$be the null generator of $\mathcal{I}^{+}$containing $q$ (the past endpoint $i^{0}$ of $\Lambda^{+}$ does not belong to $\left.\Lambda^{+}\right)$, let $\Lambda^{-}$be the null generator of $\mathcal{I}^{-}$containing $p$, and let $C(p, q)$ be the space of causal curves in $\left(\widetilde{D}, \widetilde{g}_{a b}\right)$ from $p$ to $q$.

Let $C_{0}(p, q)$ be a connected component of $C(p, q)$, and let $\gamma \in C_{0}(p, q)$ be a causal curve from $p$ to $q$ which does not meet $\Lambda^{+}$except at $q$. A curve $\gamma^{\prime} \in C_{0}(p, q)$ is "faster" if it arrives at some earlier point $q^{\prime}$ (weakly earlier: possibly $q^{\prime}=q$ ) of $\Lambda^{+}$, and then extends along $\Lambda^{+}$from $q^{\prime}$ to $q$. Let $\mathcal{A} \subseteq \Lambda^{+}$be the set of "points of arrival" on $\Lambda^{+}$of curves in $C_{0}(p, q)$; that is, $r \in \mathcal{A}$ iff there is a causal curve in $C_{0}(p, q)$ from $p$ which first meets $\Lambda^{+}$at $r$. Obviously $\mathcal{A}$ is linearly ordered and either has supremum $q$ or is empty (which occurs iff $C_{0}(p, q)$ is empty). We will argue that $\mathcal{A}$ cannot have an infimum in $\Lambda^{+}$, whence either $C_{0}(p, q)$ contains the joined-up null curve $\Lambda^{-} \cup\left\{i^{0}\right\} \cup \Lambda^{+}$or it is empty.

If instead $\mathcal{A}$ were to have an infimum $Q \in \Lambda^{+}$, then we may consider a net, indexed by $\mathcal{A}$, of curves in $C_{0}(p, q)$ whose points of arrival converge to $Q$. By the global hyperbolicity assumption, $C(p, q)$ is compact, whence so is $C_{0}(p, q)$, so this net in $C_{0}(p, q)$ converges to a limit causal curve $\Omega \in C_{0}(p, q)$ through $Q$. Since this curve is in $C_{0}(p, q)$, it does not arrive at $\Lambda^{+}$before $Q$, which implies that $\omega:=\Omega \cap \mathcal{D}$ is non-empty. Then $\omega$ joins $\Lambda^{-}$to $\Lambda^{+}$by passing through $\mathcal{D}$, so clearly it is complete, as measured by affine parameters in $\left(D, g_{a b}\right)$. Now we show that it's a line.

At this stage, a direct proof of (ii) is possible (cf. the argument sketched in [3]), but we will obtain it as a corollary of $(i)$ instead. We cannot immediately assert in this case that $Q$ (and thus $\omega$ ) lies on $\partial I^{+}(p)$, since we have not shown that timelike curves in some other component of $C(p, q)$ do not join $p$ to $Q$. However, we can assert that for $C_{0}(p, q)$ to be a connected component there must be some open set in $C(p, q)$ that contains $\Omega$ and does not contain any curve in $C(p, q) \backslash C_{0}(p, q)$. This open set is necessarily a union of basis sets $\mathcal{B}(\mathcal{O})$, so there must be a $\widetilde{D}$-open set $\mathcal{O}$ (recall $\widetilde{D}$ is the extended spacetime for $D$ ) such that $\Omega \subseteq \mathcal{O}$ and such that no element of $C(p, q) \backslash C_{0}(p, q)$ is contained as a subset of $\mathcal{O}$. It follows that $Q$ lies on the boundary of $J^{+}(p, \mathcal{O})$, the relative causal future, since any causal curve in $\mathcal{O}$ joining $p$ to $\Lambda^{+}$belongs to $C_{0}(p, q)$ and so cannot arrive on

\footnotetext{
${ }^{4}$ As alluded to earlier, the focusing lemma used for the proof of active topological censorship given by [3] does not require the generic condition, in contrast to what is needed here. There is also a subtle difference in the sorts of energy conditions that these two proofs require.
} 
$\Lambda^{+}$before $Q$. But $\left(\mathcal{O}, \tilde{g}_{a b}\right)$ is a spacetime in its own right, and in this spacetime we can assert that $Q$ lies on $\partial I^{+}(p)$, whence so does the curve $\omega$. Thus $\omega$ is a complete null line in the spacetime $\left(D \cap \mathcal{O}, g_{a b}\right)$, which contradicts the focusing assumption (which holds on $\left(D, g_{a b}\right)$, and therefore holds on the subset $\left.\left(D \cap \mathcal{O}, g_{a b}\right)\right)$. We can resolve this contradiction by dispensing with the assumption that the set $\mathcal{A}$ of arrival points for members of $C_{0}(p, q)$ has an infimum in $\Lambda^{+}$.

If $C_{0}(p, q)$ has no infimum and is non-empty, then curves in $C_{0}(p, q)$ arrive arbitrarily early (arbitrarily close to $i^{0}$ ) on $\Lambda^{+}$. Since $C_{0}(p, q)$ is compact, it must therefore contain a limit curve which passes through $i^{0}$. But only one causal curve can go from $p \in \mathcal{I}^{-}$ through $i^{0}$ to $q \in \mathcal{I}^{+}$, namely the joined-up null curve $\Lambda^{-} \cup\left\{i^{0}\right\} \cup \Lambda^{+}$. We have therefore shown that every connected component of $C(p, q)$ must contain this curve, so there can only be one connected component, proving $(i)$.

To prove $(i i)$, we simply note that if two causal curves from $p$ to $q$ are not homotopic (here we refer to homotopies on the space of all curves, not just causal curves, in $\mathcal{D}$ ), then $\mathcal{D}$ will have a universal covering space whose $\mathcal{I}$ will consist of multiple disjoint copies of the $\mathcal{I}$ of $\mathcal{D}$. Let $\mathcal{I}_{\alpha}$ and $\mathcal{I}_{\beta}$ be two distinct copies, and choose $p \in \mathcal{I}_{\alpha}^{-}$and $q \in \mathcal{I}_{\beta}^{+}$. Applying the above reasoning to any component $C_{0}(p, q)$ of $C(p, q)$, then either $C_{0}(p, q)$ is empty or there is a causal curve from $p$ that passes through $i_{\beta}^{0}$ on its way to $q$. But in the latter case such a curve could not remain entirely on $\mathcal{I}$ from $p$ to $i_{\beta}^{0}$ since $\mathcal{I}_{\alpha}$ and $\mathcal{I}_{\beta}$ are not mutually connected, and it cannot pass through spacetime to reach $i_{\beta}^{0}$ because its projection into $\mathcal{D}$ would be a curve that passes through spacetime and then reaches $i^{0}$, which is forbidden. Hence, no such curve can exist, and $C_{0}(p, q)$ must be empty. This will be true for every connected component of $C(p, q)$, so $C(p, q)$ is empty, proving ( $i i)$.

3. Interpretation. Before interpreting part $(i)$ of the theorem, it will be useful to review the interpretation of part (ii). As we have said, Gannon's singularity theorem (Corollary 1.2 of [5]) shows that incomplete null geodesics exist in physically reasonable spacetimes admitting non-simply connected Cauchy surfaces. Gannon's theorem is consistent with figure 1 (depicting the UCS of a specific example spacetime), where the horizon shields the singularity but does not prevent causal curves that begin on one component of $\mathcal{I}$ from ending on another. The point of active topological censorship is that horizons appear early enough to catch all causal curves that might otherwise "traverse the spacetime topology" and so connect different components of $\mathcal{I}$ in the UCS, as in figure 2. This provides an interpretation for the result in [3], and for Theorem 2.1(ii) above.

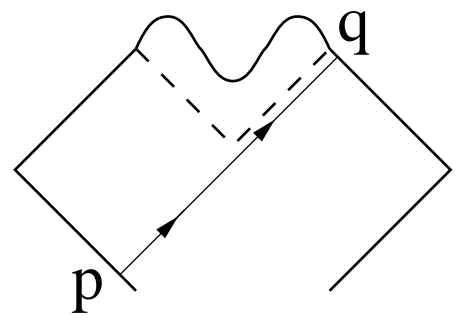

Figure 1

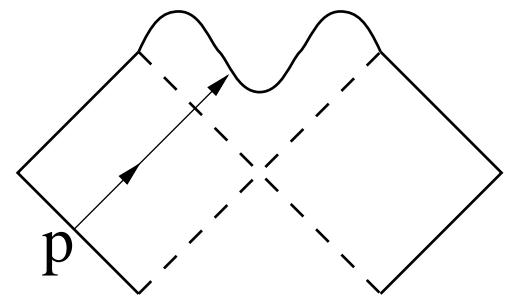

Figure 2 
Moreover, Galloway has shown [4] that the result of [3] can be extended in a direct fashion to prove that the domain of outer communications is simply connected.

In what follows, we will refer to the specific example of a black hole whose horizon intersects some spacetime Cauchy surface in a torus, although what is said will also apply if the horizon has higher genus. Topological censorship implies that every causal curve from $p \in \mathcal{I}^{-}$to $q \in \mathcal{I}^{+}$can be continuously deformed within the domain of outer communications to a causal curve from $p$ to $q$ and which is always near infinity, although the interpolating curves through which the deformation proceeds need not be causal. This eliminates the possibility of what one might call "eternal toroidal horizons" (unless they are supported by negative energy densities which cause de-focusing of null geodesics), since such spacetimes admit causal curves from $p$ to $q$ that "thread the torus" and so cannot be deformed, even acausally, to infinity without meeting the horizon. In particular, in a stationary spacetime with a toroidal black hole one could find a pair of causal curves from $p \in \mathcal{I}^{-}$to $q \in \mathcal{I}^{+}$and one could join up the pair at $p$ and at $q$ to form a single closed (but of course not future-causal) curve which would be linked with the horizon, and would violate topological censorship ${ }^{5}[2]$.

A similar argument [7] may be used to show a stronger result that toroidal horizons which are not present on some initial surface but form later cannot persist as torii into the infinite future. One may say from this that a toroidal horizon can have only a finite lifetime, and so it must "decay," presumably to spherical topology. Such a phenomenon is observed to occur in numerical evolutions of the Einstein equations [6].

This brings us to our primary interests, an interpretation for Theorem 2.1(i) and an apparent paradox which was raised in [7], where a resolution was also conjectured. Consider a domain of outer communications $\mathcal{D}$ and a time coordinate $t$ orthogonal to a system of spacetime Cauchy surfaces. ${ }^{6}$ Say that horizons are at first absent (for $\left.t<0\right)$ and then a symmetrical toroidal horizon forms about the origin (for $t \in(0, T)$ ). Let us for convenience characterize the size of the torus by $R$, the radius in some convenient coordinates of the largest coordinate circle lying on the torus. In accordance with the above discussion, say that for $t>T$ only a horizon of spherical topology is present, presumably because the toroidal horizon has expanded until it begins to self-intersect in the centre. Now fix a point $p \in \mathcal{I}^{-}$such that a null geodesic from this point passes through the origin (so this geodesic is "axial") at time $t$. Since $(t, 0,0,0)$ is not behind the horizon, this geodesic can escape back out to some $q \in \mathcal{I}^{+}$, but to observers moving along integral curves of $\partial / \partial t$, this geodesic will appear to thread through the torus.

If this curve were to thread the torus, would that contradict part $(i)$ of the theorem?

Theorem 2.1(i) falls just short of claiming that every causal curve, and in particular the curve which appears to thread the horizon above, is causally homotopic (i.e. con-

\footnotetext{
${ }^{5}$ In the context of arbitrary (as opposed to causal) curves, a linked curve usually means a closed curve that is not homotopic to a point. In consequence, if one fixes two points $p$ and $q$ on such a linked curve, the two resulting portions of the curve define curves from $p$ to $q$ that are not homotopic to each other.

${ }^{6}$ Although the parts of these surfaces that lie outside the horizon are not Cauchy surfaces for $\mathcal{D}$, we do assume global hyperbolicity of $\mathcal{D}$ in order that Theorem 2.1 applies.
} 
tinuously deformable, remaining always within $C(p, q))$ to a causal curve near infinity. The distinction is that this theorem claims only that $C(p, q)$ is connected, not arcwise connected; the latter, stronger condition is necessary to guarantee a homotopy. However, given the topology on $C(p, q)$, the former condition is sufficiently strong so as to capture the physical content of the discussion of [7]. In any case, this is really just a technical exercise; the physical arguments of [7] already clearly illustrate what is at issue here.

To proceed, let $\gamma \in C(p, q)$ and let $\mathcal{O}_{\sigma}(\gamma)$ belong to a family of "open thickenings" of $\gamma$ which shrink down on $\gamma$ as $\sigma$ gets large. Consider any collection of sets drawn from such thickenings, one for each curve in $C(p, q)$, say the set defined by $\sigma=\sigma_{0}$. This defines an open cover $\left\{\mathcal{B}\left(\mathcal{O}_{\sigma_{0}}(\gamma)\right) \mid \gamma \in C(p, q)\right\}$ for $C(p, q)$. No matter how large $\sigma_{0}$ is (hence, no matter how "thin" these "thickenings" are), this cover cannot be disjoint, since $C(p, q)$ is connected. Therefore, from any initial curve, say $\gamma_{1} \in C(p, q)$, one must be able to move to some other curve $\gamma_{2} \in C(p, q)$ such that the respective thickenings $\mathcal{B}\left(\mathcal{O}_{\sigma_{0}}\left(\gamma_{1}\right)\right)$ and $\mathcal{B}\left(\mathcal{O}_{\sigma_{0}}\left(\gamma_{2}\right)\right)$ not only intersect each other but also contain a curve $\gamma_{12} \in C(p, q)$ within this intersection. From this second curve, one must similarly be able to move to a third curve $\gamma_{3}$, and so on, until one arrives at any desired final curve, and this is possible no matter how large $\sigma_{0}$. 7

Hence there is indeed something of an apparent paradox, for which Jacobson and Venkataramani suggested the following resolution. Consider now that we deform this null geodesic off-axis and out to infinity. For deformations which move the geodesic off-axis by a coordinate amount $\sim R$ (defined above), Jacobson and Venkataramani suggest that the geodesic, in order to remain a null geodesic, also receives a "time-advance" by an amount $>T$. Then this deformed geodesic, which might otherwise be expected to encounter the horizon, arrives within and escapes from the black hole formation region before the horizon has in fact formed. Because of this time-advance mechanism associated with offaxis deformations of the geodesic, the original geodesic can in fact be causally deformed to a causal curve near infinity, in agreement with part $(i)$ of the theorem, and where we interpret the word "deform" in the sense of the preceding paragraph (i.e. through a chain, but not necessarily through a homotopy, in $C(p, q))$.

A time-advance mechanism is familiar in Schwarzschild spacetime, where off-axis null geodesics are also advanced relative to axial ones. This effect is named for Shapiro [10], who first suggested its importance as a test of relativity theory. However, the Shapiro effect is witnessed by observers at rest with respect to the timelike Killing field of Schwarzschild spacetime, and so has the necessary invariant character that gives it physical significance (hence it permits a useful experimental test of general relativity). In contrast, the time-advance discussed above is witnessed by observers moving perpendicularly to the surfaces $t=$ const. These surfaces have no differential-geometric significance, and indeed cannot be orthogonal surfaces to a Killing field. The invariant description is that no "causal linking" of a causal curve through the horizon has occurred, because in

\footnotetext{
${ }^{7}$ Moreover, essentially the same procedure can be followed even if $\sigma_{0}$, the "inverse thickness" of each thickening, varies. Note that one can go from any initial to any final curve via only finitely many such moves, even when $\sigma_{0}$ varies, because of the compactness of $C(p, q)$. The curves $\gamma_{1}, \gamma_{2}, \ldots$ are then said to comprise a chain.
} 
virtue of Theorem 2.1(i), $C(p, q)$ is connected. The absence of such causal linking is the desired interpretation of Theorem 2.1(i).

Lastly, although the notion of linking is usually referred to arcwise connectivity, we have not seen a need here to extend the theorem to assert arcwise connectivity of $C(p, q)$. Beyond perhaps yielding a clearer development of certain known results concerning variations of causal curves, the question arises as to whether a compelling motivation to investigate this extension exists, especially since it is not presently clear whether such an extension should exist. ${ }^{8}$

4. Further remarks. Finally, we discuss the interesting implications of a point raised by G. Galloway during the oral presentation of this paper. We consider now a domain of outer communications which has the property that the past-inextendible null geodesics reaching $\mathcal{I}^{+}$are past-complete, and we assume that these geodesics are governed by a focusing lemma. Let us further assume that this domain is not simply connected, so that it has a multi-sheeted covering by a universal covering space whose own $\mathcal{I}$ therefore has at least two components. Lastly, we assume that for each point $i_{\alpha}^{0}$ covering $i^{0}$, there is a neighbourhood $\mathcal{N}_{\alpha} \ni i_{\alpha}^{0}$ which is not in causal contact with $\mathcal{I}_{\beta}^{-}$for $\alpha \neq \beta$.

Now by arguments in [4], $\mathcal{I}_{\alpha}$ must be in causal contact with some $\mathcal{I}_{\beta}$, for otherwise the UCS would not be connected. But if $\mathcal{I}_{\alpha}$ and $\mathcal{I}_{\beta}$ were in causal contact but had neighbourhoods of their respective $i^{0}$ s which were not, then we could find a point $p \in \mathcal{I}_{\alpha}^{-}$ such that the boundary of the future of this point intersects $\mathcal{I}_{\beta}^{+}$(or this is true but with $\alpha$ and $\beta$ interchanged). From this point of intersection, we could trace back along a null generator of this boundary which, by standard arguments, would be an inextendible null line and, by assumption, complete (it could be past-trapped, however, and so it need not reach back to $\left.\mathcal{I}_{\alpha}^{-}\right)$. By the focusing assumption, complete null lines are forbidden, so we would have a contradiction, which is resolved if $\mathcal{I}_{\alpha}$ and $\mathcal{I}_{\beta}$ are not in causal contact after all. Since this in turn would contradict the argument of [4], it must be that $\mathcal{I}_{\alpha}$ and $\mathcal{I}_{\beta}$ cannot be distinct components of the $\mathcal{I}$ of the UCS after all, which leads in turn to the conclusion that the domain of outer communications must be simply connected.

But recall the Introduction, wherein we described how horizons cloaking the singularity served to enforce topological censorship. This description echoed the common understanding that cosmic censorship, rather than global hyperbolicity per se, is the key assumption underpinning topological censorship. Because the argument just presented relies on geodesic completeness and not on global hyperbolicity, variations of it show

\footnotetext{
${ }^{8}$ The Mazurkiewicz-Menger-Moore theorem states that every compact, connected, locally connected space is arcwise connected. That $C(p, q)$ obeys the first of these conditions follows from global hyperbolicity while, for $p \in \mathcal{I}^{-}$and $q \in \mathcal{I}^{+}$, the second follows from Theorem 2.1. The third is the condition that every neighbourhood of every curve in $C(p, q)$ has a connected subneighbourhood. Now a straightforward argument which uses only that the sets $I^{ \pm}$are open (this argument does not require $p, q \in \mathcal{I}$, nor does it require any focusing lemma or causal regularity condition to hold) suggests that timelike curves are always points of local connectivity in $C(p, q)$. However, no obvious argument presents itself as to why null geodesics, or even curves which contain a null geodesic segment, should be points of local connectivity.
} 
promise as tools with which to explore the link between cosmic and topological censorship. This issue is presently under investigation (and has recently been resolved, see [13]).

Acknowledgements. I thank Piotr Chruściel and Ted Jacobson for an e-mail exchange last year which helped me to understand this topic, and Piotr for remarks on this paper. I'd like to thank Greg Galloway for the remark cited in Section 4, and for discussions concerning the role of cosmic censorship in topological censorship theorems. I'd like to thank all of the above and Greg Burnett for discussions, and also John Friedman for an explanation of the FSW theorem in terms of conformal diagrams. I thank my collaborators in ref. [9] for permission to quote liberally from our work (specifically the proof of Theorem 2.1(i)) prior to its publication.

\section{References}

[1] A. Borde, Geodesic focusing, energy conditions and singularities, Class. Quantum Gravit. 4 (1987), 343-356.

[2] P. T. Chruściel and R. M. Wald, On the topology of stationary black holes, Class. Quantum Gravit. 11 (1994), L147-L152.

[3] J. L. Friedman, K. Schleich, and D. W. Witt, Topological censorship, Phys. Rev. Lett. 71 (1993), 1486-1489.

[4] G. Galloway, On the topology of the domain of outer communication, Class. Quantum Gravit. 12 (1995), L99-L101.

[5] D. Gannon, Singularities in nonsimply connected space-times, J. Math. Phys. 16 (1975), 2364-2367.

[6] S. A. Hughes, et al., Finding Black Holes in Numerical Spacetimes, Phys. Rev. D49 (1994), 4004-4015.

[7] T. Jacobson and S. Venkataramani, Topology of event horizons and topological censorship, Class. Quantum Gravit. 12 (1995), 1055-1061.

[8] R. Penrose, R. D. Sorkin, and E. Woolgar, A positive mass theorem based on the focusing and retardation of null geodesics, preprint gr-qc/9301015, 1993; R. Penrose Light rays near $i^{0}$ : a new mass-positivity theorem, Twistor Newsletter 30 (1990), 1-5; R. D. Sorkin and E. Woolgar, New demonstration of the positivity of mass, in: Proc. Fourth Can. Conf. on Gen. Rel. and Rel. Astrophys. , G. Kunstatter, D. E. Vincent, and J. G. Williams (eds.), World Scientific, Singapore, 1992, 206-210.

[9] R. Penrose, R. D. Sorkin, and E. Woolgar, in preparation.

[10] I. I. Shapiro, Fourth test of general relativity, Phys. Rev. Lett. 13 (1964), 789-791.

[11] R. D. Sorkin and E. Woolgar, A causal order for spacetimes with $C^{0}$ Lorentzian metrics: proof of compactness of the space of causal curves, Class. Quantum Gravit., to appear (1996) (cf. gr-qc/9508018).

[12] F. J. Tipler, Energy conditions and spacetime singularities, Phys. Rev. D17 (1978), 2521-2528; T. A. Roman, Quantum stress-energy tensors and the weak energy condition, Phys. Rev. D33 (1986), 3526-3533; On the "averaged weak energy condition" and Penrose's singularity theorem, Phys. Rev. D 37 (1988), 546-548.

[13] G. J. Galloway and E. Woolgar, The cosmic censor forbids naked topology, Class. Quantum Gravit. 14 (1997), L1-L7. 